\title{
Young leaders doing diabetes education in a school
}

\author{
Matheus Chaluppe de Oliveira, Ronaldo José Pineda Wieselberg ${ }^{*}$, Mark Thomaz Ugliara Barone, \\ Patrícia Vieira de Luca, Viviana Giampaoli \\ From 20th Brazilian Diabetes Society Congress \\ Porto Alegre, Brazil. 11-18 November 2015
}

\section{Background}

Although type 1 diabetes mellitus (DM) is one of the most common chronic diseases in children, school staff and students know very little about it. Most of what they know are misconceptions, like: "the person who has it cannot eat sugar", or "it is a disease of old people". Due to this lack of knowledge, they sometimes refuse to have students with $\mathrm{DM}$, to assist them with their self-care routine, or are unable to help in an emergency. One of the Brazilian Young Leaders in Diabetes (YLD) made, as its conclusion YLD Training project, a diabetes information campaign in his former school.

\section{Materials and methods}

In addition to the speech in 6 classrooms (126 students, grades 8th-12th, ages between 13 and 17 yrs., 44\% boys and $56 \%$ girls), 4 pamphlets were made and placed on the school dashboards (about: hypoglycemia, DM symptoms, and invitation to the speech). A questionnaire was used before and after the speeches. It contained multiple choice questions about: existence of a family member with DM; appropriate help for someone with DM who is shaking, dizzy, pale and nervous; frequency of sports practice; behaviors that help in preventing type $2 \mathrm{DM}$; if it is possible to cure DM or not; and if he/she had DM. There was also an open-ended question about DM symptoms. Wilcoxon signed rank test with the continuity correction was used to compare the pre- and post-speech number of right and wrong answers to each question.

\section{Results}

There was only 1 student with T1D. Most of them (63\%) reported to have a family member with DM (20\% no family member with DM, and $17 \%$ did not know). In addition, $49 \%$ practice physical activity 3 or more times a week, $29 \%$ only once a week, and $22 \%$ do not practice. The 4 questions compared pre- and post-speech presented significant difference in terms of higher number of correct answers after the speech, all of them with a p-value $<0.001$.

\section{Discussion}

Our objectives were partially achieved, since after the speech most of the students, but not all, answered correctly the questions about: hypoglycemia correction (percentage of right answer pre- and post-speech: $21 \%$ and $64 \%$ ), type 2 DM preventive behaviors (62\% and $78 \%$ ), diabetes symptoms (10\% and $71 \%)$, and DM possibility of cure (49\% and 91\%).

\section{Conclusion}

We believe that led by a Young Leader the project may be more effective, because the connection with someone from a closer age group and language may enhance students' interest.

Published: 11 November 2015

\section{doi:10.1186/1758-5996-7-S1-A185}

Cite this article as: de Oliveira et al:: Young leaders doing diabetes education in a school. Diabetology \& Metabolic Syndrome 2015 7(Suppl 1): A185.

\footnotetext{
* Correspondence: r.daeron@gmail.com

Young Leaders In Diabetes (IDF/ADJ), São Paulo, Brazil
} 